\title{
Reduced Prefrontal-Parietal Effective Connectivity and Working Memory Deficits in Schizophrenia
}

\author{
Lorenz Deserno, ${ }^{1}$ Philipp Sterzer, ${ }^{1,3}$ Torsten Wüstenberg, ${ }^{1}$ Andreas Heinz, ${ }^{1,2,3}$ and Florian Schlagenhauf ${ }^{1}$ \\ ${ }^{1}$ Department of Psychiatry and Psychotherapy, Campus Charité Mitte, Charité-Universitätsmedizin Berlin, and ${ }^{2}$ Cluster of Excellence NeuroCure, \\ Charite-Universitätsmedizin Berlin, 10117 Berlin, Germany, and ${ }^{3}$ Bernstein Center of Computational Neuroscience, 10115 Berlin, Germany
}

The neural mechanisms behind cognitive deficits in schizophrenia still remain unclear. Functional neuroimaging studies on working memory (WM) yielded inconsistent results, suggesting task performance as a moderating variable of prefrontal activation. Beyond regional specific activation, disordered integration of brain regions was supposed as a critical pathophysiological mechanism of cognitive deficits in schizophrenia. Here, we first hypothesized that prefrontal activation implicated in WM depends primarily on task performance and therefore stratified participants into performance subgroups. Second, in line with the dysconnectivity hypothesis, we asked whether connectivity in the prefrontal-parietal network underlying WM is altered in all patients. We used functional magnetic resonance imaging in human subjects (41 schizophrenia patients, 42 healthy controls) and dynamic causal modeling to examine effective connectivity during a WM task. In line with our first hypothesis, we found that prefrontal activation was differentially modulated by task performance: there was a significant task by group by performance interaction revealing an increase of activation with performance in patients and a decrease with performance in controls. Beyond that, we show for the first time that WM-dependent effective connectivity from prefrontal to parietal cortex is reduced in all schizophrenia patients. This finding was independent of performance. In conclusion, our results are in line with the highly influential hypothesis that the relationship between WM performance and prefrontal activation follows an inverted U-shaped function. Moreover, this study in a large sample of patients reveals a mechanism underlying prefrontal inefficiency and cognitive deficits in schizophrenia, thereby providing direct experimental evidence for the dysconnectivity hypothesis.

\section{Introduction}

Impaired working memory (WM) is among the best-established findings in schizophrenia (Lee and Park, 2005; Forbes et al., 2009). However, previous functional neuroimaging studies yielded highly controversial results with regard to regional specific activation, while a meta-analysis of these studies suggests that the neural network subserving WM is disrupted in schizophrenia (Glahn et al., 2005).

In schizophrenia patients, hypoactivations, hyperactivations, or no differences compared with healthy controls were described in dorsolateral prefrontal cortex (dlPFC) during WM (Manoach, 2003). Another meta-analysis demonstrated that this inconsistency primarily depends on task performance (Van Snellenberg et al., 2006). Hence, levels of dlPFC activation may reflect neural processing efficiency indicating individual differences of working memory capacity (Callicott et al., 1999). As a consequence, an

Received July 5, 2011; revised Oct. 6, 2011; accepted Oct. 17, 2011.

Author contributions: L.D., P.S., T.W., A.H., and F.S. designed research; L.D., P.S., T.W., A.H., and F.S. performed research; T.W. contributed unpublished reagents/analytic tools; L.D., P.S., T.W., A.H., and F.S. analyzed data; L.D., P.S., T.W., A.H., and F.S. wrote the paper.

This work was supported by a grant from the German Research Foundation (DFG HE2597/4-3 and 7-3 and DFG Exc 257) (A.H.) and by the German Ministry for Education and Research (BMBF 01QG87164 and 01GS08159). We thank Martin Dinges, Michael Koslowski, Dimitri Filonov, and Anne Beck for help during data acquisition.

The authors declare no competing financial interests.

Correspondence should be addressed to Dr. Florian Schlagenhauf, Department of Psychiatry and Psychotherapy, Charité-Universitätsmedizin Berlin, Campus Mitte Charitéplatz 1, 10117 Berlin, Germany. E-mail: florian. schlagenhauf@charite.de.

DOI:10.1523/JNEUROSCI.3405-11.2012

Copyright $\odot 2012$ the authors $\quad 0270-6474 / 12 / 320012-09 \$ 15.00 / 0$ inverted U-shaped function of dlPFC activation was proposed (Callicott et al., 2003): High-performing patients recruit dlPFC stronger than low-performing patients, but less efficiently than controls. This inverted U-curve hypothesis elegantly reconciles inconsistencies between previous studies.

It is conceivable that disrupted functional integration in the WM network explains neural inefficiency in schizophrenia more precisely and was proposed as a specific pathophysiological mechanism of cognitive impairments in schizophrenia (Friston and Frith, 1995; Andreasen et al., 1999). Electrophysiological animal research showed that interdependence of firing rates in dIPFC and parietal cortex (PC) is essential for WM performance (Chafee and Goldman-Rakic, 2000). Likewise, studies in healthy human subjects using imaging and electrophysiological methods demonstrated prefrontal-parietal functional connectivity during WM (Gazzaley et al., 2004; Sauseng et al., 2005). The dysconnectivity hypothesis of schizophrenia focuses on abnormal synaptic plasticity (Friston, 1998). Synaptic plasticity is strongly related to glutamatergic neurotransmission and its modulation by other transmitter systems (Lau and Zukin, 2007). From a theoretical perspective, this hypothesis aims to explain a common pathophysiology of the illness as well as the heterogeneity of clinical symptoms. In this context, dynamic causal modeling (DCM) of effective connectivity offers a simplistic but reasonable model of synaptic plasticity (Stephan et al., 2006, 2009a).

Supporting this hypothesis, mainly altered functional connectivity was reported in schizophrenia by neuroimaging studies with a focus on prefrontal-temporal connections (Pettersson- 
Yeo et al., 2011). Electrophysiological research in patients provides evidence for a contribution of abnormal neural oscillations to cognitive deficits (Uhlhaas and Singer, 2010). Reduced structural connectivity including the superior longitudinal fasciculus (Shergill et al., 2007; Rowland et al., 2009), which connects frontal and parietal cortices and is critically involved in WM (Karlsgodt et al., 2010), also supports the notion of dysconnectivity. However, despite the well replicated impairment of WM and a strong focus on dlPFC dysfunction in schizophrenia, direct evidence for WM-dependent alterations in effective connectivity underlying dlPFC inefficiency is still lacking.

Here, we hypothesized the following: (1) In accord with the previously proposed inverted U-curve, fMRI signal in dlPFC during a WM task would increase with performance in patients, while the opposite relationship would be observed in controls; therefore participants were stratified in performance subgroups. (2) In line with the dysconnectivity hypothesis, WM-dependent prefrontal-parietal effective connectivity would be altered in all schizophrenia patients independent of performance.

\section{Materials and Methods}

\section{Participants and instruments}

A total of 83 participants, 41 schizophrenia patients and 42 healthy controls, were included. Patients fulfilled DSM-IV and ICD-10 criteria for schizophrenia, had no other psychiatric axis I disorder (SCID interview) (First et al., 2001) and no current drug abuse or past history of drug dependence other than nicotine consumption (SCID interview and random urine drug testing). Patients were recruited at the Department of Psychiatry and Psychotherapy (Campus Charité Mitte) of the Charité Universitätsmedizin. Ten female and 31 male patients with a mean age of 34.12 years (SD, \pm 10.44$)$ were included. Thirty-five patients were treated with typical antipsychotics (haloperidol, $n=22$; flupenthixol, $n=10$; fluphenazine, $n=2$; perazine, $n=1$ ), five with atypicals (aripriprazole, $n=2$; olanzapine, $n=2$; risperidon, $n=1$ ), and one patient was unmedicated. Psychopathological symptoms were assessed with the positive and negative syndrome scale (PANSS) (Kay et al., 1987) resulting in a mean total PANSS score of $78.85 \pm 28.18$ (positive symptoms, $20.15 \pm$ 7.98; negative symptoms, $20.15 \pm 8.47$; general psychopathology, $37.83 \pm 14.99)$. The patient sample showed a mean duration of illness of $5.79 \pm 6.75$ years, a mean age of onset of $28.62 \pm 8.59$ years, and a mean number of $3.10 \pm 3.05$ episodes. Patients were treated with a mean dosage of 523.25 $\pm 316.29 \mathrm{mg}$ in chlorpromazine equivalents (Davis, 1974; Woods, 2003) and had a mean verbal IQ of $96 \pm 13.08$ as assessed with a German vocabulary test (Schmidt and Metzler, 1992) and a mean of $11 \pm$ 3.28 years of education.

Healthy controls had no axis I or II psychiatric disorder (SCID interview) (First et al., 1997, 2001), no family history of psychiatric disorders in first-degree relatives, no current drug abuse or a past history of drug dependence other than nicotine consumption (SCID interview and random urine drug testing). Nineteen female and 23 male healthy controls aged $35.36(\mathrm{SD}, \pm 12.25$ ) years were included. Healthy controls had a mean verbal IQ of $110(\mathrm{SD}, \pm 10.50)$ and a mean of 15 years of education ( $\mathrm{SD}, \pm 3.70)$. As in previous studies, verbal IQ was significantly higher in healthy controls, as were years of education (Potkin et al., 2009; Morris et al., 2011).

The local ethics committee approved the study, and after complete description of the study to the participants, written informed consent was obtained.

\section{Working memory task}

Participants underwent fMRI scanning while performing a numeric $n$-back WM task as used in previous studies (Schlagenhauf et al., 2008, 2010). The task contains two conditions: (1) in the "2-back" condition, participants were required to press a button when the number they saw equaled the number seen two numbers before; and (2) in the "0-back" condition, participants had to respond with a button press each time they saw the number zero. Numbers between 0 and 9 were displayed for 500 ms with an intertrial interval of 900 ms. Each block consisted of 22 stimuli containing three targets and was indicated by an instruction cue displayed for $2 \mathrm{~s}$ before each block. Stimulation blocks and resting periods alternated within the experiment with a total of six 2-back and six 0 -back blocks. During resting periods, volunteers were instructed to fixate on a cross in the center of the screen.

\section{Behavioral data analysis}

To analyze behavioral performance during the numeric $n$-back task, the sensitivity index $d^{\prime}$ was calculated for both conditions separately. It derives from signal detection theory and allows the distinction of signal and noise, where a higher $d^{\prime}$ indicates better signal detection. This procedure provides one measure of correct hits and false alarms for each of the conditions of the WM task. The individual probabilities of hits and false alarms were transformed into $z$-scores using the inverse cumulative distribution function in Matlab (icdf). Measures of sensitivity $\left(d^{\prime}\right)$ were computed according to Wickens (2002) as follows: $d^{\prime}=z$ (probability(hits) $)-z$ (probability(false alarms)). In this case, a higher $d^{\prime}$ indicates more accurate performance. In addition, response times were computed for the target stimuli within the 2-back and 0-back conditions. To test for differences in performance accuracy and response times, both were entered into separate mixed ANOVAs with condition as within-subject factors and group as between-subject factor. Post hoc $t$ tests were performed to clarify the direction of the results and all reported results remained significant when including age, gender, handedness, verbal IQ, and years of education as covariates.

High, middle, and low performance subgroups were separated at $33 \mathrm{rd}$ and 66th percentiles of $d^{\prime} 2$-back within each group. The three performance subgroups of schizophrenia patients did not differ significantly with regard to any characteristics: PANSS scores (all values of $p>0.28$ ), type of medication $(p=0.42)$, chlorpromazine equivalents $(p=0.19)$, age of onset $(p=0.06)$, duration of illness $(p=0.20)$, number of episodes $(p=0.32)$, verbal IQ $(p=0.30)$, or years of education $(p=0.23)$.

\section{Functional magnetic resonance imaging}

fMRI was performed on a 1.5 tesla scanner (Magnetom VISION; Siemens) equipped with a standard circularly polarized head coil (CPHeadcoil) using gradient-echo echo-planar imaging (TR, $2600 \mathrm{~ms}$; TE, $40 \mathrm{~ms}$; flip angle, $90^{\circ}$; matrix, $64 \times 64$; voxel size, $\left.4 \times 4 \times 5.5 \mathrm{~mm}\right)$. The combination of a stimulus onset asynchrony of $1400 \mathrm{~ms}(500 \mathrm{~ms}$ stimulus duration; $900 \mathrm{~ms}$ intertrial interval) (see above, Working memory task) and a TR of $2600 \mathrm{~ms}$ resulted in 13 possible time points of stimulus presentation per TR. Across multiple stimulus presentations, this yielded an effective sampling rate of $5 \mathrm{~Hz}$. Twenty-four slices approximately parallel to the bicommissural plane (anterior commissure-posterior commissure plane) were collected, covering the whole brain. Twenty fMRI volumes were acquired per block: 12 during stimulation (2-back or 0 -back) and eight during the resting period. Blocks were presented alternately three times in each of the two runs (A B A B A B). A total of 252 volumes were collected.

\section{fMRI data analysis}

fMRI data was analyzed with SPM8 (http://www.fil.ion.ucl.ac.uk/spm/). The first three volumes of each functional time series were discarded to remove non-steady-state effects caused by $\mathrm{T} 1$ saturation. Before preprocessing, the origin of the functional time series was set to the anterior commissure. The ArtRepair toolbox for SPM (http://www.fil.ion.ucl. ac.uk/spm/ext/\#ArtRepair) was used to denoise volumes that are outliers based on global intensity or large scan-to-scan movement (Mazaika et al., 2009). Volumes were realigned to a mean image of both functional time series to correct for between-scan movements. The mean image was normalized to the standard EPI template provided by the Montreal Neurological Institute (MNI), and the parameters obtained in the normalization matrix were applied to the realigned images, which were resliced with a voxel size of $4 \times 4 \times 4 \mathrm{~mm}$. Finally, all images were smoothed with a Gaussian kernel (full width at half-maximum, $8 \mathrm{~mm}$ ).

Statistical analysis was performed in the context of the general linear model (GLM) using a two-level approach (Friston et al., 1995). On the first level of analysis, condition blocks of 2-back and 0-back and the instruction cue were modeled. To account for movement-related vari- 


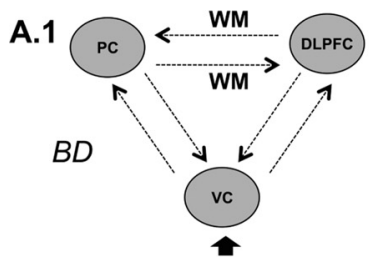

B 1)
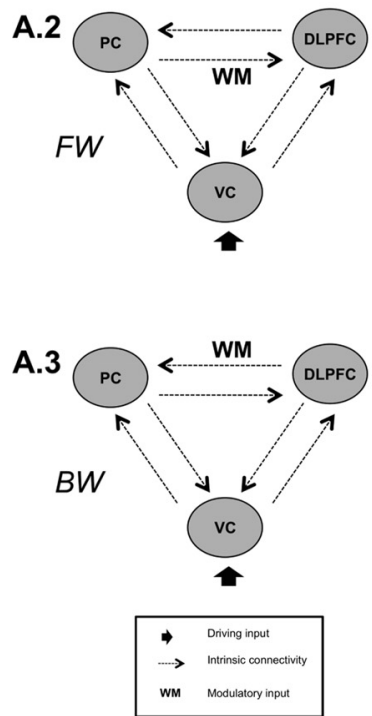

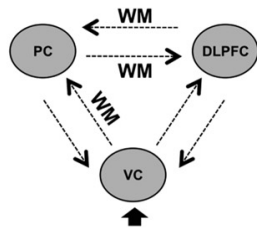

5)

6)

9)
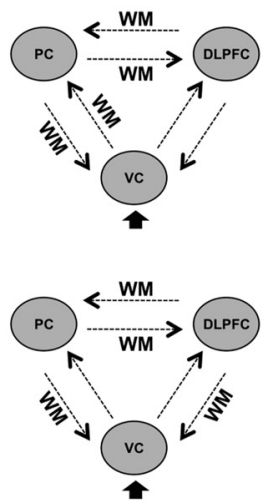

13)

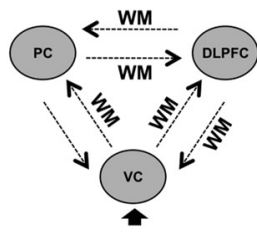

2)
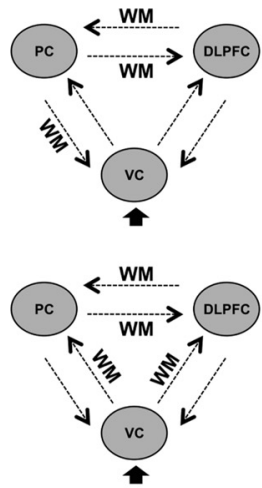

7)

10)

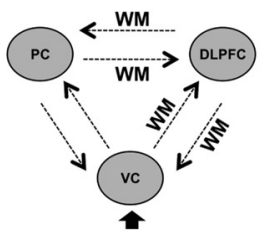

14)

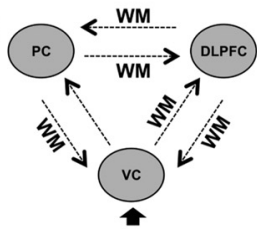

3)
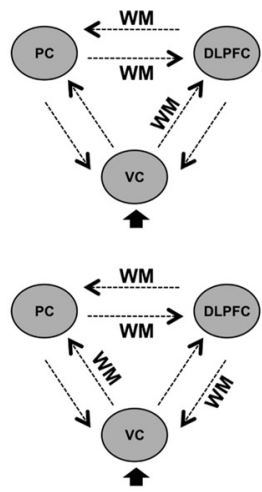

4)

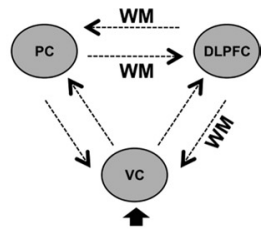

8)

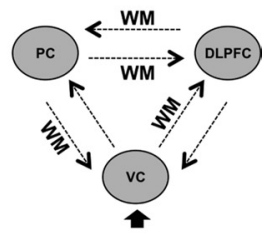

11)

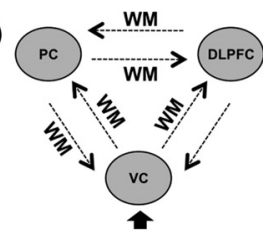

12)

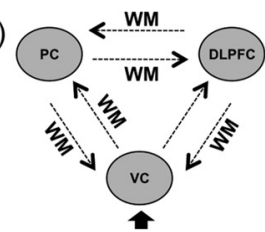

15)

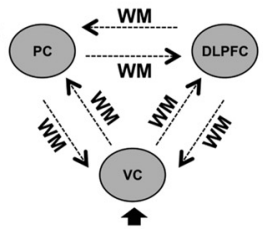

16)

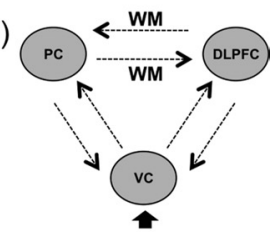

Figure 1. Model space. $A$, Initial model of prefrontal-parietal connectivity with bidirectional (BD) (A.1) modulation and the two other model families, with only forward (FW) (A.2) or backward (BW) (A.3) modulation. B, Sixteen models with additional modulations of the connections from VC to dIPFC and PC, respectively, here only shown for the BD family of models.

ance, the realignment parameters were added as regressors in the design matrix. Contrast images for 0 -back versus baseline and 2-back versus baseline were computed and taken to the second level for random-effects inference. On the second level, a mixed ANOVA with task (2-back/0back) as within-subject factor and the between-subject factors group (healthy controls/schizophrenia patients) and performance (low/middle/high) was performed. The main effect of task is reported at a significance level of $p<0.05$, FWE-corrected across the whole brain and a cluster extent of 5 voxels. The two interactions of interest (task by group and task by group by performance) are reported at $p<0.05$, FWEcorrected for the main effect of task. All reported results remained significant when including age, gender, handedness, verbal IQ, and years of education as covariates. Post hoc $t$ contrasts were computed to compare performance subgroups.

To specifically test the hypothesized quadratic relation between performance and activation in dlPFC, mean parameter estimates for the contrast "2-back > 0-back" were extracted from the main effect of task using an anatomical dlPFC mask (BA 9 and BA 46) derived from the WFU Pick Atlas toolbox (Lancaster et al., 2000; Maldjian et al., 2003) and subjected to a quadratic regression analysis.

\section{Dynamic causal modeling}

Overview. DCM10 as implemented in SPM8 was used to analyze effective connectivity. In DCM, regional time series derived from a GLM analysis are used to analyze connectivity and its modulation by experimental conditions. DCM models hidden neuronal dynamics and the influence that one neuronal system exerts over another (Friston et al., 2003). DCM allows modeling of the endogenous coupling between two regions, which is context independent ("intrinsic connections"). The impact of experimental stimuli can be modeled directly on specific regions ("driving inputs") or on the strength of coupling between two regions ("modulatory input"). In DCM, the modeled neuronal dynamics need to be transformed into a measured response, in this case the BOLD signal. This is done by using a hemodynamic forward model (Friston et al., 2000;
Stephan et al., 2007). Parameter estimation is performed in a Bayesian framework as described previously (Friston et al., 2003).

Time series extraction. Results from previous studies as well as the results of our GLM analysis motivated a focus on prefrontal-parietal connectivity. Therefore, dlPFC and PC were included. Regional time series were extracted on the single-subject level using a combination of functional and anatomical criteria: second-level analysis clusters revealed by the main effect of task of the respective regions were masked with anatomical regions of interest (ROIs) taken from the WFU Pick Atlas toolbox: BA9/46 for dlPFC and BA7/40 for PC. Visual cortex (VC) was used as a direct input region for driving inputs. A contrast comprising all visual events in the task was used to generate a VC cluster and this was again combined with an anatomical mask of BA17. Each subject's MNI coordinates of the highest $T$ value (2-back minus 0-back for dlPFC and PC; visual contrast for VC) within these combined ROIs were surrounded with a sphere of $4 \mathrm{~mm}$ and used to extract the first eigenvariate.

Model space. We assumed intrinsic connections between all of the three regions in forward and backward direction. All visual events in the task were used as driving input and the 2-back condition as modulatory input. In our initial model (Fig. 1 A.1), the driving input was directed to $\mathrm{VC}$ and the modulatory input exerted its influence on frontoparietal connections bidirectionally. This was in line with our hypothesis that prefrontal-parietal connectivity is strengthened by the WM condition. Starting from this initial model, we constructed the following model space. Because we found no hint in the literature whether the WM condition exerts its influence predominantly on one of the two directions of prefrontal-parietal connectivity, we constructed two additional model families with an effect of the experimental condition only on one of the two directions [forward model family: PC $\rightarrow$ dlPFC (Fig. 1A.2); backward model family: dlPFC $\rightarrow$ PC (Fig. 1 A.3)]. Based on the finding that activity in primary sensory cortices correlates with higher association cortices (Gazzaley et al., 2004; Kelley and Lavie, 2011), we extended the model space by all 15 possible combinations of the modulatory input 
with the intrinsic connections from and to VC resulting in 16 models (Fig. $1 B$ ). Each of the three families (bidirectional, backward, forward) contained these 16 models of modulatory effects. This resulted in a total of 48 models, which were computed for both hemispheres. To find one best model and to compare families of models, Bayesian model selection (BMS) was used as described below.

Bayesian model selection. A comparison of the above-described model space was performed using BMS to identify a model that fits the data of both groups best. This allows testing for between-group differences on optimal model parameters using classical inference. For BMS, we used a novel random-effects approach for group studies (Stephan et al., 2009b). BMS takes into account the fit of the models, primarily based on the number of free parameters, in relation to the model complexity. With higher complexity, the relative fit of a model may increase but generalizability may be reduced. Random-effects BMS gives so-called exceedance probabilities, the probability that one model is more likely than another. The BMS version integrated in DCM10 for SPM8 also integrates BMS on family levels to identify families of models, which are more likely than others (Penny et al., 2010).

First, BMS was conducted treating healthy controls and schizophrenia patients as one group. As between-group differences in estimated parameters may be due to differences in model fit, BMS was also performed for both groups separately. If model fit differs between groups on the level of a specific model or on a family level, this constitutes a result in its own right. In addition, Bayesian model averaging (BMA) is an alternative approach to allow for statistical comparison of parameters (Stephan et al., 2010). BMA provides weighted averages of the entire model space or a family of models where the weighting is given by the posterior probability for each model (Penny et al., 2010). As pointed out in detail in methodological work by Penny et al. (2010), a marginal posterior is calculated for subject $n$ and family $k$ to provide the weighting. Thus, the influence of models with a low posterior probability to the estimation of the marginal posterior is low.

Group statistic of DCM parameters. Classical inference for randomeffects inference on parameters of a best model or averaged parameters of a model subspace requires correction for multiple comparisons. Therefore, results of two-sample $t$ tests are reported at a Bonferronicorrected statistical threshold $p<0.0045$. Results that were significant at an uncorrected threshold of $p<0.05$ are reported as trends. All results remained significant when conducting ANCOVAs with age, gender, handedness, verbal IQ, and years of education as covariates. Group by performance ANOVAs were performed to test the influence of performance on connectivity.

\section{Results}

\section{Behavioral performance}

ANOVA with sensitivity index $d^{\prime}$ revealed a significant main effect of task $(F=262.82 ; \mathrm{df}=1 ; p<0.001)$, a significant main effect of group $(F=47.13 ; \mathrm{df}=1 ; p<0.001)$, and no significant task by group interaction $(F=0.09 ; \mathrm{df}=1 ; p=0.77)$. This indicated impaired performance in schizophrenia patients (SZ) compared with healthy controls (HC) (post hoc t test HC $>$ SZ for 2-back: $t=4.87, p<0.001$; and for 0-back: $t=4.85, p<0.001$ ) and a better performance during the 0 -back condition compared with the 2-back condition (post hoc paired $t$ tests for HC: $t=$ $16.43, p<0.001$; SZ: $t=12.33, p<0.001)$.

ANOVA with response times revealed a significant main effect of task $(F=62.05 ; \mathrm{df}=1 ; p<0.001)$, a significant main effect of group $(F=13.38 ; \mathrm{df}=1 ; p<0.001)$, and no significant task by group interaction $(F=0.09 ; \mathrm{df}=1 ; p=0.77)$. This indicated slower reaction times in patients compared with controls (post hoc $t$ test SZ $>$ HC for 2-back: $t=3.60, p=0.002$; and for 0-back: $t=3.24, p<0.001)$ and slower reaction times during 2-back compared with the 0 -back condition (post hoc paired $t$ tests for HC: $t=9.28, p<0.001$; SZ: $t=4.23, p<0.001)$.

\section{fMRI results}

There was a significant main effect ( $p<0.05$, whole brain FWEcorrected) of task in the WM network including bilateral superior frontal gyrus, middle frontal gyrus, medial frontal gyrus, orbital gyrus, cingulate gyrus, superior parietal lobule, superior temporal gyrus, cerebellum, and left inferior frontal gyrus in healthy controls and schizophrenia patients, which included our main regions of interest, PC and dIPFC (Table 1, Fig. 2A). There was no significant task by group interaction in dIPFC or PC, neither at a whole-brain corrected threshold nor when restricting smallvolume FWE correction to those voxels that showed a significant main effect of task. Likewise, even a less conservative approach with small-volume FWE correction within single prefrontal and parietal ROIs did not reveal any significant task by group interaction. The task by group interaction reached significance in orbitofrontal cortex (rectal gyrus), an area that exhibited reduced fMRI signal during WM compared with the control condition (0-back) (Table 1). In patients, this signal reduction during the WM condition was less pronounced.

In line with our hypothesis, the group by task by performance interaction reached significance (FWE corrected for voxels showing a main effect of task) in superior frontal gyrus and middle frontal gyrus corresponding to the right dlPFC, the left ventrolateral prefrontal cortex (vlPFC) as well as bilateral premotor cortex (Table 1; Fig. $2 B$, top panel). This group by task by performance interaction $(F=13.23 ; x=62, y=16, z=38)$ was due to an increase of activation with WM performance in schizophrenia patients (SZ high $>$ SZ low: $T=4.72$ ), while it decreases with performance in healthy controls ( $\mathrm{HC}$ low $>\mathrm{HC}$ high: $T=2.47$ ) (Fig. $2 B$, middle panel).

To specifically test the hypothesis of an inverted U-curve of dlPFC activation, mean parameter estimates for the contrast " 2 back - 0-back" derived from an anatomical dlPFC mask were subjected to a quadratic regression. Across all participants, patients and controls, this analysis revealed the hypothesized effect of a quadratic association for 2-back $d^{\prime}$ predicting dlPFC parameter estimates $(b=-0.40 ; T=-2.21 ; p=0.048)$. The model tested explained a significant proportion of variance $\left(R^{2}=0.085\right.$; $F=3.74 ; \mathrm{df}=2 ; p=0.028$.

\section{DCM results}

Bayesian model selection

We first used BMS to compare the model evidence for the three families of models with either bidirectional, forward, or backward modulation of prefrontal-parietal connections. The model family of backward modulation clearly outperformed the two other families: exceedance probability (EP) of $83.72 \%$ for the right and $98.37 \%$ for the left hemisphere when testing both groups together (Fig. 3A.1,A.2). A clear superiority was found in healthy controls alone (EP: $86.21 \%$ right hemisphere; $95.14 \%$ left hemisphere) and in schizophrenia patients while the EP was slightly decreased for the right hemisphere (EP: $61.37 \%$ right hemisphere; $96.09 \%$ left hemisphere). Comparison of single models within the backward family yielded more variable results (Fig. 3B.1,B.2). In healthy controls, Models 6 and 3, with additional modulation of the connections from VC to PC and from VC to dlPFC, were identified as the best fitting models in healthy controls for the right and the left hemisphere (EP: 60.63\% right hemisphere; $63.82 \%$ left hemisphere). In schizophrenia patients, the same models were identified for the left hemisphere, although with decreased EPs around $40 \%$. For the right hemisphere, BMS showed ambiguous results in the patient group: There were only 
Table 1. Regional brain activation during working memory

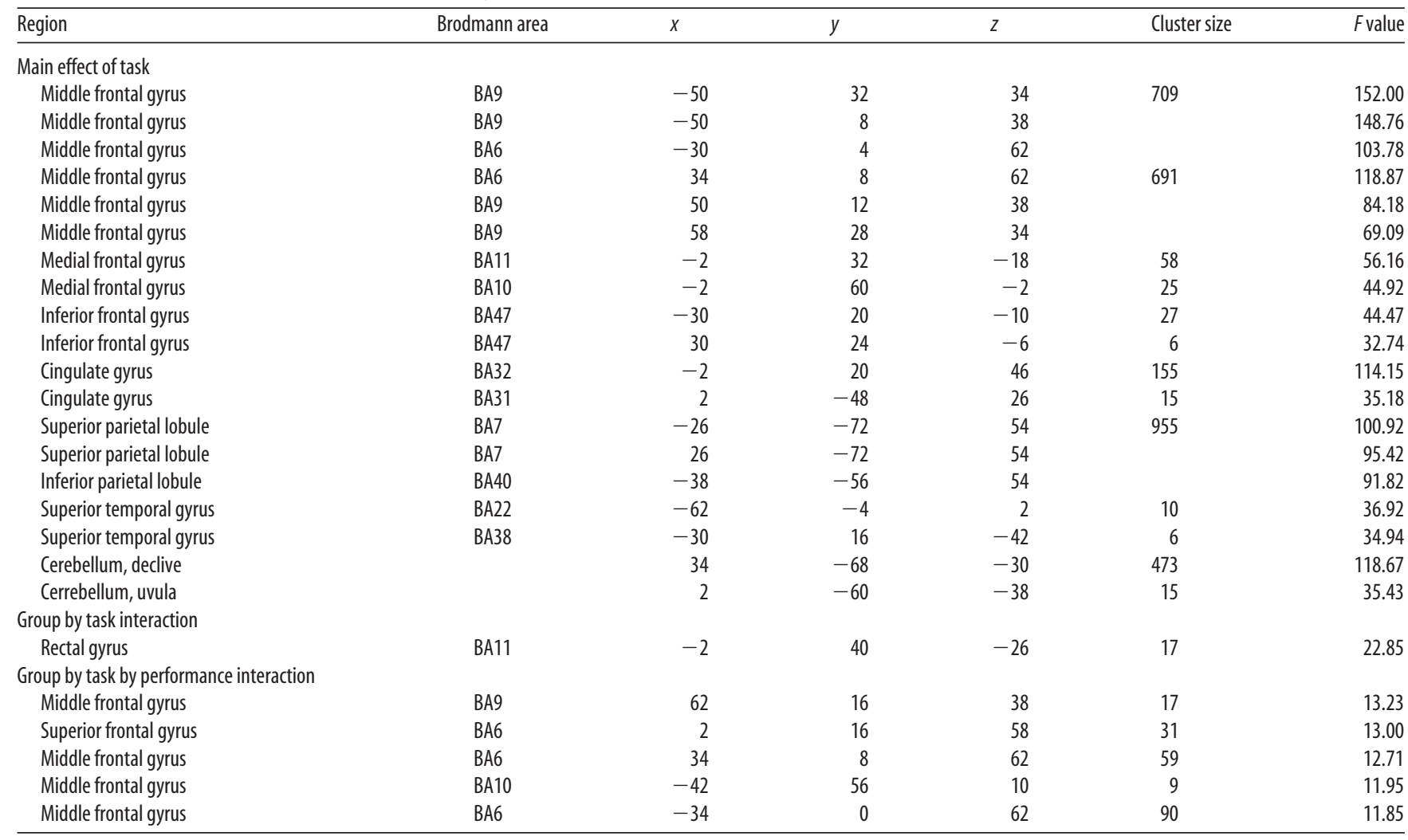

Reported are regions that show a significant WM activation for schizophrenia patients and controls taken together as indicated by the main effect of task (at $p<0.05$ whole-brain FWE-corrected) and regions that display a group by task interaction (at $p<0.05$, FWE-corrected for the main effect of task) and regions with a significant task by group by performance interaction (at $p<0.05$, FWE-corrected for the main effect of task), cluster extent $>5$ voxels.

marginal preferences for single models (all EPs $<20 \%$ ). Thus, the model fit seemed to be highly variable within the patient group.

\section{Group statistic of DCM parameters}

Due to these inconsistencies in model selection between groups, we decided to perform BMA, to avoid potentially false-positive results due to biased model selection. BMA was performed within the family of backward-modulation models to extract parameters for between-group testing. In our final group-level analysis of effective connectivity, we were able to test for differences in five modulatory parameters and six parameters of intrinsic connectivity (Fig. 4). In addition to this parameter extraction, BMA was also performed for the complete model space because patients showed a slightly lower EP for the backward family than healthy controls in the right hemisphere. This analysis confirmed the results of BMA within the family of backward-modulation models. We therefore only report results derived from the latter BMA.

Except the backward connections from PC and dlPFC to $\mathrm{VC}$ and their modulation, all other intrinsic connections and modulatory effects showed a positive influence and reached Bonferroni-corrected significance in healthy controls using onesample $t$ tests (Table 2). No significant negative parameters were observed in both groups at the Bonferroni-corrected threshold or when using an uncorrected threshold. In the right hemisphere, two-sample $t$ tests revealed significantly (Bonferroni-corrected for multiple comparisons) stronger WM-dependent modulation of connectivity from dlPFC to PC and from VC to PC in healthy controls compared with schizophrenia patients. There was also a trend toward stronger modulation of connectivity from VC to dlPFC ( $p<0.05$, uncorrected) (Table 2, Fig. 4). Intrinsic connectivity (e.g., WM-independent connectivity) was significantly (Bonferroni-corrected) stronger for connections from $\mathrm{VC}$ to
dlPFC, and from PC to dlPFC in healthy controls compared with schizophrenia patients and with an uncorrected threshold for dlPFC to PC (Table 2, Fig. 4). There were no significant group differences on connectivity parameters for the left hemisphere (all values of $p>0.14$ ). There was no significant group by performance interaction on the DCM parameters (all values of $p>$ 0.17 ) and no significant correlation with verbal IQ or years of education with the parameters of prefrontal-parietal connectivity (all values of $p>0.18$ ).

\section{Discussion}

This study demonstrates for the first time that reduced WMdependent effective connectivity from dlPFC to PC is a shared characteristic within a large sample of schizophrenia patients while alterations in functional specialization of dlPFC were only detectable when stratifying participants into performance subgroups. WM-dependent modulation of effective connectivity from dlPFC to PC was reduced in schizophrenia patients as well as the modulation of connectivity from VC to PC. These results corroborate the notion of disordered prefrontal-parietal connectivity as a core pathophysiological feature of schizophrenia. They therefore support the dysconnectivity hypothesis, which proposes altered functional integration and synaptic plasticity as a crucial mechanism in the pathophysiology of cognitive deficits in schizophrenia (Stephan et al., 2006, 2009a).

\section{Prefrontal activation and cognitive performance}

Our findings reconcile seemingly contradictory results from previous WM studies by showing that prefrontal activation reflects individual differences in cognitive performance and thus follows the previously proposed inverted U-curve (Callicott et al., 2003; 


\section{A WM activation in $\mathrm{HC}$}

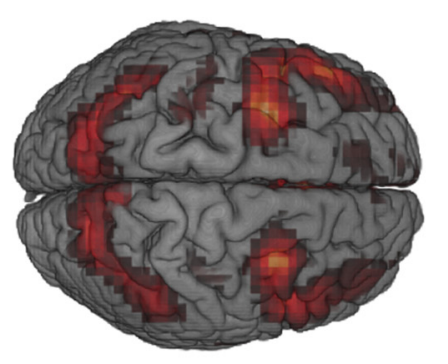

50

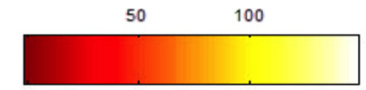

WM activation in SZ

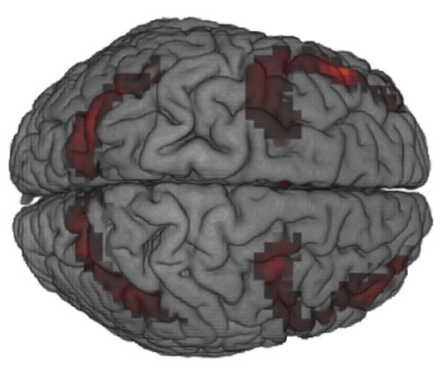

B Group $x$ task $x$ performance interaction
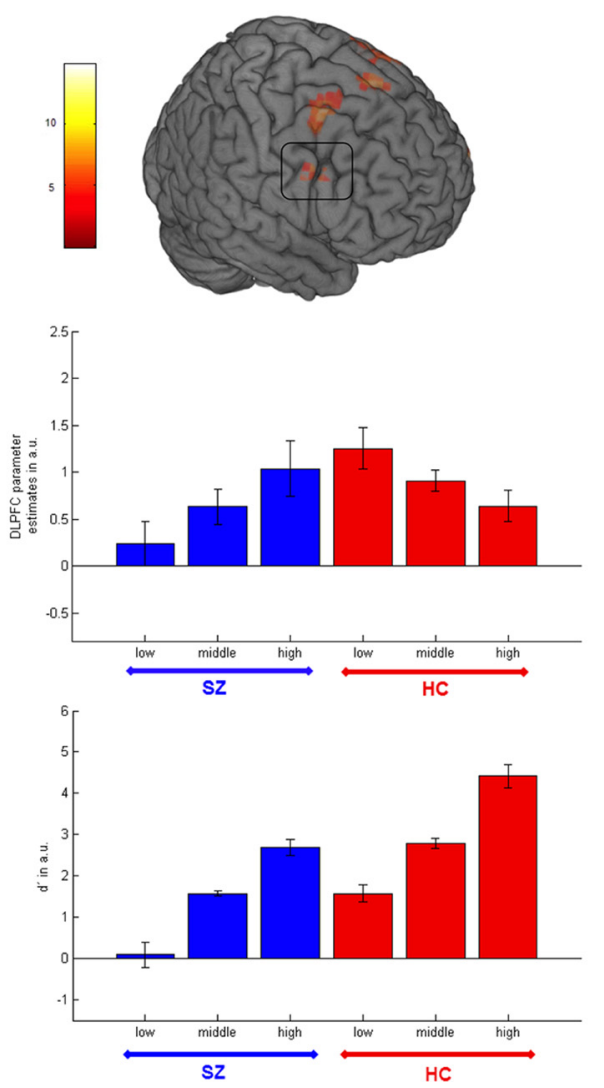

Figure 2. Regional brain activation during working memory. A, Task by group by performance ANOVA: Activation for 2-back versus 0 -back in healthy controls (top panel) and schizophrenia patients (bottom panel), indicating no significant overall differences between both groups. $\boldsymbol{B}$, Significant task by group by performance interaction in dIPFC in top panel; plot of parameter estimates for 2-back minus 0-back contrast extracted at the local dIPFC maximum within an anatomical mask of BA9 and $46[x=$ $46, y=8, z=38$ ] of the main effect of task using a $12 \mathrm{~mm}$ sphere (middle panel); plot of performance accuracy using $\mathrm{d}^{\prime}$ (bottom panel); higher performance accuracy in patients is associated with stronger dIPFC activation while activation decreases with performance in healthy controls, indicating higher efficiency in healthy controls resulting in an inverted U-curve. the authors suggest a relationship to altered activation of the default mode network during cognitive tasks (PomarolClotet et al., 2010).

\section{The dIPFC within the WM network}

Across both groups, BMS revealed that the family of models with WM-dependent modulation of the backward connection from dIPFC to PC fits the data of all participants best. This finding underlines the crucial role of dlPFC in driving functional integration in the WM network. This is in line with a recent fMRI study demonstrating that rule updating preferentially activates prefrontal cortex and stimulus updating activates $\mathrm{PC}$ in healthy participants (Montojo and Courtney, 2008). The $n$-back task used in our study requires participants to update rules, 2 -back versus 0 -back, as well as continuous stimulus updating of numbers presented within each block. In accordance, we found WMdependent modulation only from dIPFC to PC while from PC to dlPFC WM-independent intrinsic connectivity reached significance in controls. Due to the simple block design, we were not able to separate the influence of stimulus updating from rule updating on connectivity. However, it is likely that rule updating explains the presence of the WM modulation on dIPFC to PC. This interpretation is also consistent with research suggesting that the critical role of dIPFC in WM is to control activity in other task-related brain regions (D'Esposito, 2007).

In healthy participants, models with Potkin et al., 2009). That is, prefrontal activation increases with WM performance in schizophrenia patients, while it decreases in healthy controls. To the best of our knowledge, our study is the first to provide direct evidence for the inverted U-curve relationship within a large sample of patients and controls. This relationship between performance and activation reached significance in right dlPFC, left vlPFC, and bilateral premotor cortex. Remarkably, the same lateralization pattern was found in a previous study comparing performance subgroups of patients and controls (Tan et al., 2006) and is additionally supported by a meta-analysis indicating a strong performance-activation relationship in right dlPFC (Van Snellenberg et al., 2006).

Previous studies indicate that this relationship is mediated by dopamine: while WM capacity correlated positively with dopamine synthesis in healthy subjects (Cools et al., 2008), the latter predicted dlPFC activation during WM in prodromal subjects (Fusar-Poli et al., 2010).

In contrast to this clear and robust performance-dependent effect, there was no general group difference with respect to WMrelated activation in the prefrontal-parietal network. A significant task by group interaction was only observed in orbitofrontal cortex. This region showed stronger activation during the 0 -back condition when compared with the 2-back condition and this effect was diminished in patients. This is in accord with a recent study describing the same finding as "a failure to deactivate" and additional WM-dependent modulation on connections from VC to PC and to dlPFC were identified as best fitting models. High levels of attention may drive these effects, thus enabling strengthened stimulus updating during WM. Especially PC was implicated in number representation (Eger et al., 2003). Therefore, this effect might result from higher attention to possible WM targets during the 2-back condition. It could not be explained by a modulation of backward connectivity from dlPFC or PC to VC or by higher $\mathrm{VC}$ activity during WM blocks in the univariate analysis. A considered (Stephan et al., 2008).

\section{Dysconnectivity and cognitive deficits}

In line with previous research, the present study found that regional specific activation of the prefrontal cortex during WM strongly relies on cognitive performance and is inefficient in schizophrenia patients. Our study goes clearly beyond this observation by revealing a mechanism potentially underlying cognitive deficits in schizophrenia; that is, the reduced influence that dlPFC exerts over PC during WM. This effect was independent of performance and elucidates a mechanism behind dlPFC inefficiency in schizophrenia. Thus, reduced control of dlPFC on activation in PC may be responsible for shifting schizophrenia patients to the left side of the inverted U-curve. As indicated in previous research and our study (Tan et al., 2006; Van Snellendirect influence of a single region on these connections might be 

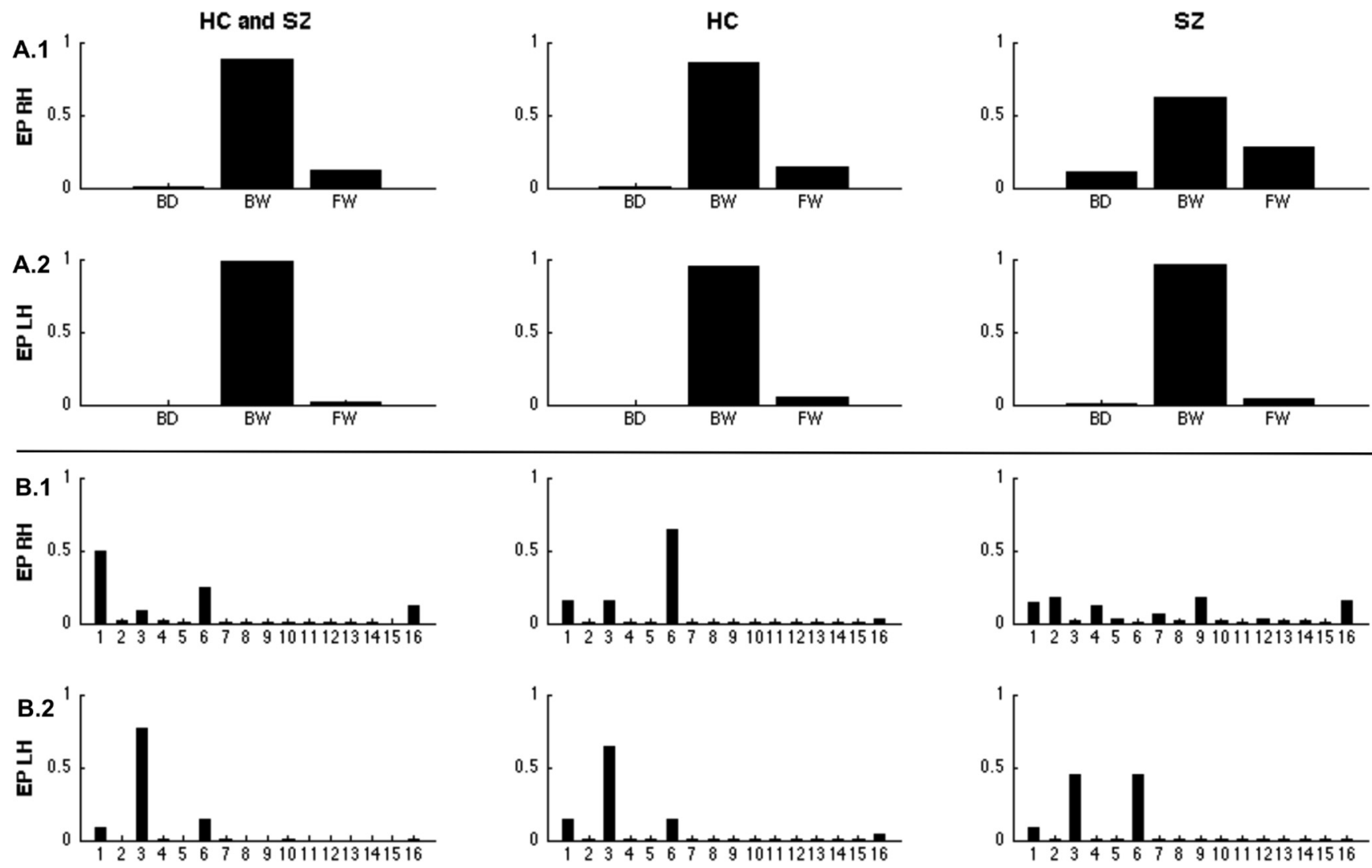

Figure 3. Results of Bayesian model selection. BMS was performed on two levels (top and bottom panels) and for healthy controls (HC) and schizophrenia patients (SZ) together (left column) and for the two groups separately (middle and right column) as well as for the right hemisphere (RH, top parts of both panels) and the left hemisphere (LH, bottom parts of both panels). Exceedance probabilities (EP) are reported as a measure of relative model fit. $A$, Family selection of bidirectional (BD), forward (FW), or backward (BW) modulation of prefrontal-parietal connectivity for the right (A.1) and the left hemisphere (A.2). B, Model selection for the BW model-subspace containing 16 models, all of them with WM modulation from dIPFC to PC and the remaining 15 possible combinations of 2-back modulation on the other connections, $\boldsymbol{B} . \boldsymbol{1}$ for the right hemisphere and $\boldsymbol{B} .2$ for the left hemisphere.

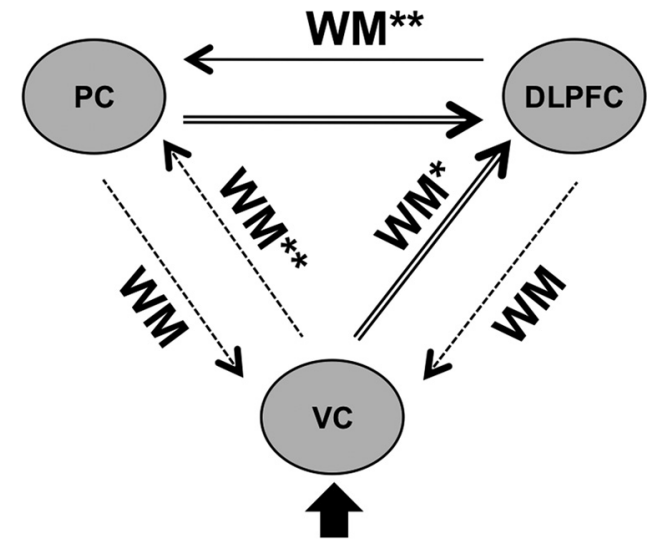

Figure 4. Group comparison of DCM parameters in the right hemisphere. Bayesian model averaging was performed for the backward subspace because of between-group differences in model selection (Fig. 3A.1); double asterisks or double arrows indicate significant Bonferronicorrected group differences, and single asterisk or single arrow indicates significant group differences uncorrected for multiple comparisons; all group differences are due to reduced parameters in schizophrenia patients.

berg et al., 2006), the inverted U-curved relationship of activation and performance was observed in right dIPFC and this lateralization was mirrored in our DCM analysis.

DCM allows to model altered synaptic plasticity (Stephan et al., 2006, 2009a). There is evidence that this alteration is related to a glutamatergic hypofunction of NMDA receptor signaling, es-
Table 2. DCM parameters

\begin{tabular}{|c|c|c|c|c|}
\hline & \multirow[b]{2}{*}{ HC } & \multirow[b]{2}{*}{$S Z$} & \multicolumn{2}{|c|}{$\begin{array}{l}\text { Two-sample } t \text { test for } \\
\text { group comparison }\end{array}$} \\
\hline & & & $T$ value & $p$ value \\
\hline \multicolumn{5}{|l|}{ Modulatory effects } \\
\hline $2 \mathrm{~b}$ on VC $\rightarrow \mathrm{PC}$ & $0.52(0.77)^{* *}$ & $0.08(0.38)$ & 3.28 & $<0.0045^{* *}$ \\
\hline $2 \mathrm{~b}$ on VC $\rightarrow$ dIPFC & $0.25(0.49)^{* *}$ & $0.02(0.18)$ & 2.85 & $0.0062^{*}$ \\
\hline $2 \mathrm{~b}$ on $\mathrm{PC} \rightarrow \mathrm{VC}$ & $-0.01(0.03)$ & $0.01(0.20)$ & -0.56 & 0.5670 \\
\hline $2 \mathrm{~b}$ on $\mathrm{dIPFC} \rightarrow \mathrm{VC}$ & $0.01(0.05)$ & $0.01(0.06)$ & 0.17 & 0.8625 \\
\hline $2 \mathrm{~b}$ on $\mathrm{dIPFC} \rightarrow \mathrm{PC}$ & $0.46(0.55)^{* *}$ & $-0.02(0.13)$ & 5.46 & $<0.0045^{* *}$ \\
\hline \multicolumn{5}{|l|}{ Intrinsic connectivity } \\
\hline $\mathrm{VC} \rightarrow \mathrm{PC}$ & $0.13(0.18)^{* *}$ & $0.07(0.16)^{*}$ & 1.70 & 0.0946 \\
\hline $\mathrm{VC} \rightarrow \mathrm{dIPFC}$ & $0.20(0.28)^{* *}$ & $0.05(0.13)^{*}$ & 3.00 & $<0.0045^{* *}$ \\
\hline $\mathrm{PC} \rightarrow \mathrm{VC}$ & $-0.01(0.10)$ & $0.03(0.07)^{*}$ & -1.79 & 0.0763 \\
\hline $\mathrm{PC} \rightarrow \mathrm{dIPFC}$ & $0.19(0.21)^{* *}$ & $0.04(0.08)^{*}$ & 4.30 & $<0.0045^{* *}$ \\
\hline $\mathrm{dIPFC} \rightarrow \mathrm{VC}$ & $-0.01(0.12)$ & $0.03(0.05)^{*}$ & -1.92 & 0.0592 \\
\hline $\mathrm{dIPFC} \rightarrow \mathrm{PC}$ & $0.13(0.17)^{* *}$ & $0.05(0.11)^{*}$ & 2.68 & $0.0091^{*}$ \\
\hline
\end{tabular}

Connectivity parameters of the right hemisphere for schizophrenia patients (SZ) and healthy controls (HC): means of parameters derived from Bayesian model averaging with standard deviations in brackets. Within each group, $1 t$ tests, and for group comparisons, $2 t$ tests were used. For the latter, $t$ and $p$ values are reported.

**Bonferroni's correction for multiple comparisons at $p<0.0045$; *uncorrected threshold, $p<0.05$.

pecially in dlPFC (Laruelle et al., 2003; Lewis and GonzalezBurgos, 2006). Therefore, our finding could be interpreted as a consequence of NMDA receptor hypofunction. This interpretation also takes into account previous research that reported a dopaminergic hypofunction in dlPFC (Okubo et al., 1997) and its relationship to impaired working memory performance (AbiDargham et al., 2002), as well as findings of subcortical hyperdo- 
paminergic neurotransmission (Breier et al., 1997; Abi-Dargham et al., 1998). Here, DCM enabled us to identify the direction of disrupted WM-dependent corticocortical dynamics contributing to dlPFC inefficiency and cognitive deficits in schizophrenia.

Previous studies using methods of effective connectivity did not examine task-dependent changes in connectivity during WM, either due to methodological issues (Schlösser et al., 2003a,b) or did not analyze WM-dependent changes when using DCM (Benetti et al., 2009; Crossley et al., 2009). The latter studies focused on the direction of task-independent intrinsic connectivity and are in line with early observations of altered temporalprefrontal correlations. As demonstrated in the present study, the analysis of WM-dependent modulation of connectivity helps to reveal a potential mechanism underlying cognitive deficits.

\section{Compensatory effects within the prefrontal cortex}

As in the present study, Tan et al. (2006) compared performance subgroups of patients and controls. With respect to the relationship of activation and performance in right dlPFC and left vlPFC, they reported similar results and additionally assessed functional connectivity using seed voxel analysis. They found stronger coupling between dlPFC and PC in controls and stronger coupling of vlPFC and PC in patients. This was interpreted as a compensatory effect in patients. The influence of vlPFC activation on prefrontal-parietal connectivity could be assessed in future studies using nonlinear DCM (Stephan et al., 2008). Anyway, such compensatory effects are in line with the dysconnectivity hypothesis that does not simply equal lower connectivity as indicated by disconnectivity, but rather stronger connectivity can be part of dysfunctionally altered connectivity (Stephan et al., 2009a).

\section{Limitations}

As in most of the previous WM studies, patients were treated with antipsychotic medication. The absence of an overall group difference in the WM network makes unspecific effects of medication on hemodynamic reactivity unlikely, and we observed no significant correlation of chlorpromazine equivalents or clinical status with cognitive performance, BOLD-fMRI, or connectivity measures. Therefore, both are unlikely to account for the observed task by performance interaction in patients. In previous WM studies, impairments of encoding, retrieval, and information manipulation were demonstrated to be differentially involved in schizophrenia (Tan et al., 2005; Schlösser et al., 2008; Potkin et al., 2009). The blocked $n$-back task used in this study does not allow for a distinction of WM subprocesses.

\section{Conclusion and outlook}

Together, this study gives evidence for disordered prefrontalparietal effective connectivity in schizophrenia using DCM, demonstrating for the first time that WM-dependent modulation of effective connectivity from dlPFC to PC is reduced in patients. In conclusion, WM-dependent prefrontal-parietal dysconnectivity seems to be a common feature of schizophrenia patients pointing toward a specific mechanism underlying cognitive deficits. That is, dlPFC inefficiently controls activation in PC during WM. As the present study demonstrates within one large sample, group differences in prefrontal functional specialization are mainly driven by cognitive performance, in contrast to prefrontalparietal dysconnectivity, which appears to constitute a comprehensive characteristic of schizophrenia.

In the future, multimodal imaging may help to elucidate the underlying neurochemical mechanisms. Furthermore, animal research indicates that an interaction of dopamine and NMDA receptor activation (Tseng and O'Donnell, 2004) might be affected by antipsychotic medication (Centonze et al., 2004). This could be an interesting target for future translational pharmacological research, which might help to identify specific factors underlying the variability of treatment responses. Based on this study, future research may prove reduced WM-dependent prefrontal-parietal connectivity as a potential diagnostic marker.

\section{References}

Abi-Dargham A, Gil R, Krystal J, Baldwin RM, Seibyl JP, Bowers M, van Dyck CH, Charney DS, Innis RB, Laruelle M (1998) Increased striatal dopamine transmission in schizophrenia: confirmation in a second cohort. Am J Psychiatry 155:761-767.

Abi-Dargham A, Mawlawi O, Lombardo I, Gil R, Martinez D, Huang Y, Hwang DR, Keilp J, Kochan L, Van Heertum R, Gorman JM, Laruelle M (2002) Prefrontal dopamine $D_{1}$ receptors and working memory in schizophrenia. J Neurosci 22:3708-3719.

Andreasen NC, Nopoulos P, O'Leary DS, Miller DD, Wassink T, Flaum M (1999) Defining the phenotype of schizophrenia: cognitive dysmetria and its neural mechanisms. Biol Psychiatry 46:908-920.

Benetti S, Mechelli A, Picchioni M, Broome M, Williams S, McGuire P (2009) Functional integration between the posterior hippocampus and prefrontal cortex is impaired in both first episode schizophrenia and the at risk mental state. Brain 132:2426-2436.

Breier A, Su TP, Saunders R, Carson RE, Kolachana BS, de Bartolomeis A, Weinberger DR, Weisenfeld N, Malhotra AK, Eckelman WC, Pickar D (1997) Schizophrenia is associated with elevated amphetamine-induced synaptic dopamine concentrations: evidence from a novel positron emission tomography method. Proc Natl Acad Sci U S A 94:2569-2574.

Callicott JH, Mattay VS, Bertolino A, Finn K, Coppola R, Frank JA, Goldberg TE, Weinberger DR (1999) Physiological characteristics of capacity constraints in working memory as revealed by functional MRI. Cereb Cortex 9:20-26.

Callicott JH, Mattay VS, Verchinski BA, Marenco S, Egan MF, Weinberger DR (2003) Complexity of prefrontal cortical dysfunction in schizophrenia: more than up or down. Am J Psychiatry 160:2209-2215.

Centonze D, Usiello A, Costa C, Picconi B, Erbs E, Bernardi G, Borrelli E, Calabresi P (2004) Chronic haloperidol promotes corticostriatal longterm potentiation by targeting dopamine D2L receptors. J Neurosci 24:8214-8222.

Chafee MV, Goldman-Rakic PS (2000) Inactivation of parietal and prefrontal cortex reveals interdependence of neural activity during memoryguided saccades. J Neurophysiol 83:1550-1566.

Cools R, Gibbs SE, Miyakawa A, Jagust W, D’Esposito M (2008) Working memory capacity predicts dopamine synthesis capacity in the human striatum. J Neurosci 28:1208-1212.

Crossley NA, Mechelli A, Fusar-Poli P, Broome MR, Matthiasson P, Johns LC, Bramon E, Valmaggia L, Williams SC, McGuire PK (2009) Superior temporal lobe dysfunction and frontotemporal dysconnectivity in subjects at risk of psychosis and in first-episode psychosis. Hum Brain Mapp 30:4129-4137.

Davis JM (1974) Dose equivalence of the antipsychotic drugs. J Psychiatr Res 11:65-69.

D’Esposito M (2007) From cognitive to neural models of working memory. Philos Trans R Soc Lond B Biol Sci 362:761-772.

Eger E, Sterzer P, Russ MO, Giraud AL, Kleinschmidt A (2003) A supramodal number representation in human intraparietal cortex. Neuron 37:719-725.

First MB, Spitzer RL, Gibbon M, Williams J (1997) Structured Clinical Interview for DSM-IV Personality Disorders (SCID-II). Washington, DC: American Psychiatric Press.

First MB, Spitzer RL, Gibbon M, Williams J (2001) Structured Clinical Interview for DSM-IV-TR Axis I Disorders, Research Version, Patient Edition with Psychotic Screen (SCID-I/P W/ PSY SCREEN). New York: New York State Psychiatric Institute

Forbes NF, Carrick LA, McIntosh AM, Lawrie SM (2009) Working memory in schizophrenia: a meta-analysis. Psychol Med 39:889-905.

Friston KJ (1998) The disconnection hypothesis. Schizophr Res 30:115-125.

Friston KJ, Frith CD (1995) Schizophrenia: a disconnection syndrome? Clin Neurosci 3:89-97.

Friston KJ, Holmes AP, Worsley KJ, Poline JP, Frith CD, Frackowiak RSJ 
(1995) Statistical parametric maps in functional imaging: a general linear approach. Hum Brain Mapp 2:189-210.

Friston KJ, Mechelli A, Turner R, Price CJ (2000) Nonlinear responses in fMRI: the Balloon model, Volterra kernels, and other hemodynamics. Neuroimage 12:466-477.

Friston KJ, Harrison L, Penny W (2003) Dynamic causal modelling. Neuroimage 19:1273-1302.

Fusar-Poli P, Howes OD, Allen P, Broome M, Valli I, Asselin MC, Grasby PM, McGuire PK (2010) Abnormal frontostriatal interactions in people with prodromal signs of psychosis: a multimodal imaging study. Arch Gen Psychiatry 67:683-691.

Gazzaley A, Rissman J, D’Esposito M (2004) Functional connectivity during working memory maintenance. Cogn Affect Behav Neurosci 4:580-599.

Glahn DC, Ragland JD, Abramoff A, Barrett J, Laird AR, Bearden CE, Velligan DI (2005) Beyond hypofrontality: a quantitative meta-analysis of functional neuroimaging studies of working memory in schizophrenia. Hum Brain Mapp 25:60-69.

Karlsgodt KH, Kochunov P, Winkler AM, Laird AR, Almasy L, Duggirala R, Olvera RL, Fox PT, Blangero J, Glahn DC (2010) A multimodal assessment of the genetic control over working memory. J Neurosci 30:8197-8202.

Kay SR, Fiszbein A, Opler LA (1987) The positive and negative syndrome scale (PANSS) for schizophrenia. Schizophr Bull 13:261-276.

Kelley TA, Lavie N (2011) Working memory load modulates distractor competition in primary visual cortex. Cereb Cortex 21:659-665.

Lancaster JL, Woldorff MG, Parsons LM, Liotti M, Freitas CS, Rainey L, Kochunov PV, Nickerson D, Mikiten SA, Fox PT (2000) Automated Talairach atlas labels for functional brain mapping. Hum Brain Mapp $10: 120-131$

Laruelle M, Kegeles LS, Abi-Dargham A (2003) Glutamate, dopamine, and schizophrenia: from pathophysiology to treatment. Ann N Y Acad Sci 1003:138-158.

Lau CG, Zukin RS (2007) NMDA receptor trafficking in synaptic plasticity and neuropsychiatric disorders. Nat Rev Neurosci 8:413-426.

Lee J, Park S (2005) Working memory impairments in schizophrenia: a meta-analysis. J Abnorm Psychol 114:599-611.

Lewis DA, Gonzalez-Burgos G (2006) Pathophysiologically based treatment interventions in schizophrenia. Nat Med 12:1016-1022.

Maldjian JA, Laurienti PJ, Kraft RA, Burdette JH (2003) An automated method for neuroanatomic and cytoarchitectonic atlas-based interrogation of fMRI data sets. Neuroimage 19:1233-1239.

Manoach DS (2003) Prefrontal cortex dysfunction during working memory performance in schizophrenia: reconciling discrepant findings. Schizophr Res 60:285-298.

Mazaika P, Hoeft F, Glover GH, Reiss AL (2009) Methods and software for fMRI analysis for clinical subjects. Paper presented at the 15th Annual Meeting of the Organization for Human Brain Mapping, San Francisco, CA, June.

Montojo CA, Courtney SM (2008) Differential neural activation for updating rule versus stimulus information in working memory. Neuron 59:173-182.

Morris RW, Vercammen A, Lenroot R, Moore L, Langton JM, Short B, Kulkarni J, Curtis J, O’Donnell M, Weickert CS, Weickert TW (2011) Disambiguating ventral striatum fMRI-related bold signal during reward prediction in schizophrenia. Mol Psychiatry. Advance online publication. Retrieved November 8, 2011. doi:10.1038/mp.2011.75.

Okubo Y, Suhara T, Suzuki K, Kobayashi K, Inoue O, Terasaki O, Someya Y, Sassa T, Sudo Y, Matsushima E, Iyo M, Tateno Y, Toru M (1997) Decreased prefrontal dopamine D1 receptors in schizophrenia revealed by PET. Nature 385:634-636.

Penny WD, Stephan KE, Daunizeau J, Rosa MJ, Friston KJ, Schofield TM, Leff AP (2010) Comparing families of dynamic causal models. PLoS Comput Biol 6:e1000709.

Pettersson-Yeo W, Allen P, Benetti S, McGuire P, Mechelli A (2011) Dysconnectivity in schizophrenia: where are we now? Neurosci Biobehav Rev 35:1110-1124.

Pomarol-Clotet E, Canales-Rodríguez EJ, Salvador R, Sarró S, Gomar JJ, Vila F, Ortiz-Gil J, Iturria-Medina Y, Capdevila A, McKenna PJ (2010) Medial prefrontal cortex pathology in schizophrenia as revealed by convergent findings from multimodal imaging. Mol Psychiatry 15:823-830.

Potkin SG, Turner JA, Brown GG, McCarthy G, Greve DN, Glover GH, Manoach DS, Belger A, Diaz M, Wible CG, Ford JM, Mathalon DH, Gollub R, Lauriello J, O’Leary D, van Erp TG, Toga AW, Preda A, Lim KO
(2009) Working memory and DLPFC inefficiency in schizophrenia: the FBIRN study. Schizophr Bull 35:19-31.

Rowland LM, Spieker EA, Francis A, Barker PB, Carpenter WT, Buchanan RW (2009) White matter alterations in deficit schizophrenia. Neuropsychopharmacology 34:1514-1522.

Sauseng P, Klimesch W, Schabus M, Doppelmayr M (2005) Fronto-parietal EEG coherence in theta and upper alpha reflect central executive functions of working memory. Int J Psychophysiol 57:97-103.

Schlagenhauf F, Wüstenberg T, Schmack K, Dinges M, Wrase J, Koslowski M, Kienast T, Bauer M, Gallinat J, Juckel G, Heinz A (2008) Switching schizophrenia patients from typical neuroleptics to olanzapine: effects on BOLD response during attention and working memory. Eur Neuropsychopharmacol 18:589-599.

Schlagenhauf F, Dinges M, Beck A, Wüstenberg T, Friedel E, Dembler T, Sarkar R, Wrase J, Gallinat J, Juckel G, Heinz A (2010) Switching schizophrenia patients from typical neuroleptics to aripiprazole: effects on working memory dependent functional activation. Schizophr Res 118:189-200.

Schlösser R, Gesierich T, Kaufmann B, Vucurevic G, Hunsche S, Gawehn J, Stoeter P (2003a) Altered effective connectivity during working memory performance in schizophrenia: a study with fMRI and structural equation modeling. Neuroimage 19:751-763.

Schlösser R, Gesierich T, Kaufmann B, Vucurevic G, Stoeter P (2003b) Altered effective connectivity in drug free schizophrenic patients. Neuroreport 14:2233-2237.

Schlösser RG, Koch K, Wagner G, Nenadic I, Roebel M, Schachtzabel C, Axer M, Schultz C, Reichenbach JR, Sauer H (2008) Inefficient executive cognitive control in schizophrenia is preceded by altered functional activation during information encoding: an fMRI study. Neuropsychologia 46:336-347.

Schmidt K-H, Metzler P (1992) Wortschatztest (WST). Weinheim, Germany: Beltz Test GmbH.

Shergill SS, Kanaan RA, Chitnis XA, O’Daly O, Jones DK, Frangou S, Williams SC, Howard RJ, Barker GJ, Murray RM, McGuire P (2007) A diffusion tensor imaging study of fasciculi in schizophrenia. Am J Psychiatry 164:467-473.

Stephan KE, Baldeweg T, Friston KJ (2006) Synaptic plasticity and dysconnection in schizophrenia. Biol Psychiatry 59:929-939.

Stephan KE, Weiskopf N, Drysdale PM, Robinson PA, Friston KJ (2007) Comparing hemodynamic models with DCM. Neuroimage 38:387-401.

Stephan KE, Kasper L, Harrison LM, Daunizeau J, den Ouden HE, Breakspear M, Friston KJ (2008) Nonlinear dynamic causal models for fMRI. Neuroimage 42:649-662.

Stephan KE, Friston KJ, Frith CD (2009a) Dysconnection in schizophrenia: from abnormal synaptic plasticity to failures of self-monitoring. Schizophr Bull 35:509-527.

Stephan KE, Penny WD, Daunizeau J, Moran RJ, Friston KJ (2009b) Bayesian model selection for group studies. Neuroimage 46:1004-1017.

Stephan KE, Penny WD, Moran RJ, den Ouden HE, Daunizeau J, Friston KJ (2010) Ten simple rules for dynamic causal modeling. Neuroimage 49:3099-3109.

Tan HY, Choo WC, Fones CS, Chee MW (2005) fMRI study of maintenance and manipulation processes within working memory in first-episode schizophrenia. Am J Psychiatry 162:1849-1858.

Tan HY, Sust S, Buckholtz JW, Mattay VS, Meyer-Lindenberg A, Egan MF, Weinberger DR, Callicott JH (2006) Dysfunctional prefrontal regional specialization and compensation in schizophrenia. Am J Psychiatry 163:1969-1977.

Tseng KY, O'Donnell P (2004) Dopamine-glutamate interactions controlling prefrontal cortical pyramidal cell excitability involve multiple signaling mechanisms. J Neurosci 24:5131-5139.

Uhlhaas PJ, Singer W (2010) Abnormal neural oscillations and synchrony in schizophrenia. Nat Rev Neurosci 11:100-113.

Van Snellenberg JX, Torres IJ, Thornton AE (2006) Functional neuroimaging of working memory in schizophrenia: task performance as a moderating variable. Neuropsychology 20:497-510.

Wickens TD (2002) Elementary signal detection theory. Oxford, New York: Oxford UP.

Woods SW (2003) Chlorpromazine equivalent doses for the newer atypical antipsychotics. J Clin Psychiatry 64:663-667. 